\title{
Desafíos de la Unidad de Investigación del Instituto Nacional de Neurología y Neurocirugía
}

\section{Achievements and Challenges of the Research Unit of the National Institute of Neurology and Neurosurgery}

\author{
Ma. Guadalupe Nava Galán
}

$\mathrm{E}$ 1 surgimiento de la investigación en enfermería inició con los registros detallados de las observaciones de Florencia Nightingale, quien aplicó sus conocimientos al estudio de la realidad a través de la historia y la filosofía. Esto constituyó el centro de su vida, es decir, el cuidado de la salud de las personas. Ella analizó la lógica del pensamiento de enfermería, las bases filosóficas, epistemológicas y éticas a través de su teoría, a partir del análisis del contexto histórico sociocultural en el cual se desarrolló su principal obra. Sus bitácoras están plasmadas a través de sus notas - éstas parten de la lógica del pensamiento Nightingaleano - las cuales han servido de punto de partida para desarrollar todas y cada una de las actuales teorías de enfermería. Sobre todo, Florencia Nightingale merecen el profundo reconocimiento de haber sido la pionera del pensamiento científico, así como de cuestiones éticas en enfermería y del desarrollo disciplinar actual. ${ }^{1}$

Por las consideraciones anteriores, cabe destacar que en la evolución de la investigación de enfermería en América sus aportaciones son reconocidas desde los años cincuenta, al fundar la primera Asociación de Enfermería - la primera revista de investigación - la cual se publicó y difundió en la misma década; para los años sesenta, las prioridades de investigación y los objetivos se orientaron a la práctica, la educación y el desarrollo curricular, sin embargo, en los setenta fue este última el que tuvo mayor impulso para el desarrollo de la investigación. En los años ochenta se estableció el Centro Nacional para la Investigación de Enfermería (CNIE), el cual se considera una de las primeras unidades de investigación en enfermería; fue hasta los noventa cuando se planteó la necesidad de incorporar hallazgos de la investigación a la práctica clínica. ${ }^{2}$

Así mismo, Espinoza-Ortega refiere que los avances de enfermería en EE.UU. se han dado por etapas, las cuales son:

Primera etapa. Diferenciación de la enfermería como ciencia. Aquí se describe qué estudia la enfermería, su dominio, enfoque, límites y por qué es diferente de otras disciplinas. Segunda etapa. Legitimidad de la enfermería como academia. En esta etapa se definen las características de la teoría y se formulan criterios para evaluarla.

Tercera etapa. En ésta se considera la base de los conocimientos científicos de enfermería, centrándose en la interrogante sobre las teorías que puedan guiar la investigación.

De igual manera los mismos autores refieren que la Investigación de la enfermería en México está basada en las siguientes temáticas de estudio: el $40 \%$ refiere la formación de los recursos humanos, el $32 \%$ los factores que influyen en la práctica profesional, el $18 \%$ la asistencia de la enfermería en el diagnóstico, el 14\% la administración, el 12\% la asistencia en la promoción, mantenimiento y prevención y el $8 \%$ la asistencia de la enfermería en el tratamiento y la recuperación. En cuanto a las publicaciones se reportan, las siguientes: publicaciones clínicas 54\%, epidemiológicas $20 \%$, de servicios de salud $17 \%$ y educativas $9 \%$.

Desde entonces el perfil de las investigaciones de enfermería en México está enfocado principalmente en la

Correspondencia: Ma. Guadalupe Nava Galán. Insurgentes Sur 3877, Colonia La Fama, Delegación Tlalpan, C.P. 14269

E-mail: magpengalan@hotmail.com

Este artículo puede ser consultado en versión completa en http://www.medigraphic.com/enfermerianeurologica 
asistencia, la calidad de la atención y los aspectos educativos y de administración. En cuanto a los diseños de investigación, éstos son de tipo descriptivos en $30 \%$, no especificados en $27 \%$ y de otro tipo en $17 \%$; también $12 \%$ tipo correlaciónales y $5 \%$ cuasi experiméntales. En cuanto a las poblaciones y áreas de investigación en México se enfocan más al adulto joven, adulto mayor, adolescentes, niños y mujeres.

Las áreas de interés son las enfermedades neurológicas, las neuroquirúrgicas y las neuropsiquiátricas, así como las áreas crónicas, los estados críticos, las drogas lícitas, la calidad de vida, el autocuidado, la autoestima, el crecimiento y el desarrollo y agotamiento, entre otros. En el ámbito Internacional, la investigación en enfermería tiene como finalidad el desarrollo del conocimiento y la validación de lo ya existente, como consecuencia se genera la ciencia propia de cada disciplina; esto permite validar su práctica profesional. ${ }^{3}$ Así mismo, Moreno-Casbas enfatiza que la investigación se caracteriza por la utilización del método científico y la obtención de respuestas; él refiere también, que no es una actividad de élite, ni un lujo, sino una necesidad y, por lo tanto, debe formar parte del quehacer cotidiano de todo profesional. Es un deber de la enfermería reflexionar sobre la realidad en la que actúa, obtener conocimientos y aplicarlos. La profesionalización va más allá de marcar competencias o remarcar su identidad; ser profesional, significa poner al servicio de la sociedad la producción y uso responsable del conocimiento.

En los últimos 10 años, las instituciones los hospitales las escuelas y las facultades donde se desarrollan los profesionales de enfermería impulsan la investigación desde donde se encuentren; si cuentan con unidades destinadas a la investigación de enfermería la desarrollarán y, con asesoría para dar inicio a los protocolos, estos son asesorados, registrados y evaluados por pares.

La investigación debe surgir de las necesidades en la clínica, en la gestión y la educación, dando continuidad a las líneas establecidas por los profesionales interesados en llevar a cabo el proceso en las diferentes metodologías, sin perder el objeto de estudio («el cuidado») desde diferentes enfoques. Lo que se pretende al hacer investigación es que los resultados sean propositivos para intervenir oportunamente y que sean competitivos en diferentes niveles de atención a nivel interdisciplinar, a través de la práctica clínica, gestión y enseñanza, no sólo nacional sino internacional. ${ }^{4}$

Manfredi destaca la importancia que tienen las unidades de investigación; éstas permiten desarrollar la exploración de la enfermería en América Latina para poder conocer qué está permeando en la práctica clínica, en la educación y la gestión. La creación de unidades específicas de enfermería que basan su investigación en evidencia, identifican las siguientes ventajas: a) clarificación de metas de educación, b) identificación de vacíos en los planes de estudios y c) facilitación para desarrollar sistemas de evaluación de los programas educativos entre otros. ${ }^{5}$

Así mismo, la Organización Mundial de la Salud (OMS) en el año 2003, puntualizó que la investigación es una de las cinco prioridades para el desarrollo de la práctica de enfermería y, además puntualizó sobre la necesidad de establecer una base disciplinar de evidencia para la práctica de enfermería; ${ }^{6}$ propuso como estrategia, desarrollar un trabajo colaborativo entre organizaciones de enfermería pertenecientes a diferentes países. Esta idea fue también propuesta en la VII Conferencia Iberoamericana de Educación en Enfermería. ${ }^{7}$

En este sentido, es necesario impulsar la investigación en el ámbito local para incrementar el conocimiento que permita comparar y considerar las particularidades de los diferentes países. La investigación es necesaria para hacer uso de los recursos en forma eficiente sin afectar la calidad, al tener una definición clara de las prioridades en la producción de conocimiento de enfermería, de tal forma, que respondan tanto a la necesidad de fundamentar la ciencia de enfermería, como a las necesidades en salud. ${ }^{8}$

En Chile, el Ministerio de Educación se preocupó por desarrollar proyectos de renovación curricular con el propósito de generar profesionales capacitados con una metodología para el desarrollo curricular comparable, considerando las competencias y teniendo en cuenta la autonomía de cada profesión, para proponer así, profesionales con estudios de postgrado que sean capaces de desarrollar una metodología investigativa en la que se basen las denominadas competencias genéricas de habilidades y destrezas, las cuales buscan información, conocimiento, comunicación, trabajo en equipo, liderazgo, competencias específicas y, sobre todo, competencias necesarias para cada profesión. ${ }^{9}$

La creación de Unidades de Investigación en Enfermería es primordial para desarrollar, evaluar y difundir el conocimiento de enfermería; éstas constituyen una parte trascendental de la práctica clínica, la enseñanza y la gestión del cuidado. El propósito fundamental es beneficiar directamente a los enfermos y brindar una óptima calidad de atención a través de la investigación. La enfermería puede documentar el costo efectividad de los cuidados basados en evidencia. ${ }^{10}$

Por las consideraciones anteriores, las unidades de investigación son fundamentales para que la práctica clínica de enfermería brinde una atención basada en evidencia; esto asegurará una cuidado de la calidad costo-efectivo y es, a la vez, un distintivo de la enfermería profesional; ${ }^{11}$ 
actualmente es necesario llevar acabo investigación en torno a los enfermos que se les brinda un cuidado con recursos escasos. ${ }^{12^{*}}$ Es necesario que los profesionales de enfermería identifiquen y utilicen la mejor evidencia en su ejercicio profesional cuando falte la evidencia o ésta sea débil; deben ser capaces de generar el conocimiento necesario y fortalecer a la disciplina, implementando estrategias con pocos recursos. ${ }^{13}$ Actualmente es necesario impulsar la investigación en el ámbito donde se desarrolle cada profesional de enfermería para incrementar el conocimiento que permita considerar las particularidades de los diferentes países. Asimismo se debe hacer uso de los recursos de forma eficiente, sin afectar la calidad de la atención, teniendo las prioridades en la producción de conocimiento de enfermería, de tal forma que respondan a la necesidad de fundamentar la ciencia de la enfermería de acuerdo con las necesidades en salud. ${ }^{14}$ De igual forma la Organización Panamericana de la Salud (OPS) identifica los valores y las competencias en el profesional de enfermería, definiendo la competencia de acuerdo con lo propuesto por Kofi Annan, Secretario General de las Naciones Unidas, como «aquellas destrezas, conocimientos, conductas y características necesarias para lograr un buen desempeño en el ámbito laboral». Él destacó de igual manera la importancia de tomar en cuenta la cultura y el contexto para identificar las competencias. ${ }^{15}$

Las competencias son conocimientos, habilidades y actitudes que a) afectan el rol o responsabilidad en el trabajo, b) corresponden con la realización del trabajo, c) pueden ser medidas con estándares aceptados y d) pueden ser mejoradas mediante el entrenamiento y el desarrollo. ${ }^{16}$

Las unidades de investigación en enfermería deben ser consideradas como «la fuente de desarrollo de conocimiento» que da la característica de disciplina y que están presentes en la práctica clínica y en el desarrollo de la administración y la educación, dando respuesta a las necesidades sociales que están relacionadas con los procesos de salud-enfermedad, con las condiciones y con la calidad de vida de las personas, familias y comunidades.

Actualmente, hacia el siglo XXI, emergieron tres temas generales para investigar lo que el profesional de enfermería y los estudiantes deben enfocarse, esto es, en lo siguiente: 1) principios y leyes que gobiernan los procesos de la vida, bienestar y funcionamiento óptimo de los seres humanos enfermos o sanos; 2) patrones de comportamiento humano y su interacción con el medio ambiente en situaciones

\footnotetext{
* Lamelas EF. La investigación en el currículum de enferemería. Grado de influencia y límites de la enfermería basada en evidencia. Enfermería Clínica. 2002; 1 (4): 182-187.
}

críticas de la vida, y 3 ) procesos por los cuales se afectan los campos positivos del estado de salud. ${ }^{17}$ Sin embargo, en sus artículos, Donaldson y Bottorff proponen -de acuerdo con lo que se localiza en la producción investigativa de Colombia, Latinoamérica y el mundo- que hay todavía una gran distancia frente a las líneas propuestas. Los trabajos desarrollados según el CIE, y las prioridades de investigación en enfermería para el presente son:

1) Generar conocimiento disciplinar, que se relacione con el desarrollo y aplicación de modelos conceptuales y teorías de enfermería.

2) Desarrollar estrategias para mejorar los programas de promoción de la salud y prevención de la enfermedad, en especial, las que corresponden con la determinación de factores de riesgo, incluyendo la vigilancia y control de los mismos.

3) Establecer características de las poblaciones y evaluar las estrategias encaminadas a la modificación de factores de riesgo, puesto que se han dinamizado y transformado en los últimos años.

4) Analizar aspectos relacionados con la prestación de servicios de salud, dentro de lo cual se sugiere mejorar la calidad del cuidado de enfermería y estudiar el impacto de las intervenciones de enfermería en las metas de salud y bienestar.

5) Analizar la calidad de vida laboral de las enfermeras, la retención, la movilidad local, regional e internacional, la satisfacción laboral y el estrés laboral, así como la relación de enfermeras con la población, entre otros.

6) Participar en el desarrollo de políticas encaminadas a la equitativa, oportuna y eficiente prestación de servicios de salud.

7) Integrar la práctica basada en la evidencia, lo cual dinamiza y amplía los horizontes de cuidado, puesto que surge desde escenarios distintos y promueve el trabajo en equipo entre la docencia y la asistencia. Alrededor de estos planteamientos existe una mayor sincronía en torno a los trabajos desarrollados por las enfermeras en la producción nacional e internacional.

En el ámbito nacional, los planes curriculares de los diferentes niveles de formación deberían organizarse para exponer a los alumnos a situaciones que los impulsen a adquirir una actitud de cuestionamiento de la realidad, así como una actitud de rigor científico e interés por la verdad. Al incorporar estrategias que desarrollen el espíritu científico desde el primer semestre de la carrera, se construye un terreno digno de crédito, para que en los niveles de formación superior, los alumnos, ya con una actitud indagatoria y un interés científico, adquieran los 
conocimientos y habilidades necesarios para hacer de la investigación parte de la formación escolar y, para que la apliquen en el ámbito laboral insertando la cultura de la investigación formal e institucional. ${ }^{18}$ Es importante destacar que para dirigir una unidad de investigación se debe contar con el perfil y la experiencia disciplinar y escolaridad de postgrado, pues esto asegura que el dirigente posea el dominio y los conocimientos para formar y capacitar nuevos investigadores y así, cumplir con los objetivos propuestos en los programas de trabajo. ${ }^{19}$

Los programas de trabajo deberán contar con un organigrama, una misión y una visión; deben basarse en líneas de investigación, y éstas a su vez, en las áreas de interés que van vinculándolos en un mismo eje temático. Por ejemplo, con respecto a los profesionales de enfermería del Instituto Nacional de Neurología y Neurocirugía, sus áreas de interés son las neurociencias y su eje temático es el cuidado que debe incluir metodología cuantitativa o cualitativa con actividades de investigación realizadas por diferentes grupos que muestren resultados visibles en su producción de protocolos que sean propositivos.

Así mismo, las líneas de investigación deberán corresponder a un área del conocimiento lo suficientemente amplia para que no agote los temas de interés inherentes, sobre todo, considerando que se requiere de un trabajo interdisciplinario y multidisciplinario, así como transdisciplinario; esto va a permitir diferentes puntos de vista respecto de un mismo aspecto, sobre todo, si se tiene coherencia en torno a una orientación central, lo que permite así, el surgimiento de nuevas líneas de investigación. ${ }^{20}$

Cabe destacar que estas unidades de investigación deberán contar con un área específica que cuenten con recursos humanos y materiales para desarrollar los protocolos de investigación y, éstos a su vez, basarse en formatos institucionales ya establecidos; sobre todo que sean evaluados para enriquecer la investigación desde diferentes sugerencias o desde la evaluación del rigor metodológico. Es importante, también, que los resultados se den a conocer en foro, cartel o en una publicación, para dar respuesta a las necesidades que originaron la investigación de salud; lo ideal es que de las unidades de investigación surjan sus propias revistas.

Para desarrollar la investigación se debe contar en las instituciones de salud con el perfil y personal específico para generar nuevos recursos humanos, para ello $-\mathrm{y}$ a pesar de contar con una formación básica en el área- la investigación debe ser la vía por la cual se evidencie y guíe la práctica del cuidado. En este sentido el planteamiento de los lineamientos básicos para la creación de espacios donde se propicie ésta, se han convertido en una necesidad que deberá consolidarse de manera natural como requisito indispensable y como la formación prioritaria de los profesionales de la enfermería, ya que se requiere establecer desafíos y culminarlos como logros en al ámbito institucional. No se debe perder de vista claramente que las Unidades de Investigación son espacios en los cuales se debe llevar a cabo proyectos disciplinares basados en las necesidades institucionales y, éstos deben ser dirigidos por profesionales de enfermería que apoyen el cuidado desde el punto de vista clínico, educativo y administrativo que innoven las líneas de investigación.

La profesión de enfermería en los últimos años ha sido una de las disciplinas de mayor desarrollo en el proceso de profesionalización; éste es uno de los elementos medibles que reflejan los resultados de diferentes respuestas ante una necesidad social urgente de salud al ser investigados.

Actualmente existen indicadores en las unidades de investigación que reportan un número considerable de protocolos que se están desarrollando, en el pregrado y en las áreas clínicas. Las nuevas generaciones de profesionistas con estudios de postgrado a cargo de las unidades de investigación en las instituciones han generado nuevos resultados e investigaciones y han dado respuesta a las problemáticas planteadas al desarrollar protocolos que generen impacto al dar a conocer la problemática con resultados finales en foro, carteles y en publicaciones de las revistas que se desarrollan y dirigen por los responsables las unidades.

Éstos son sin duda datos tangibles de la transformación y grado de alcance de la enfermería actual, sin embargo, el mayor reflejo del desarrollo de las (os) enfermeros de cualquier institución debe soportarse con la evidencia científica aplicada a la calidad de atención de las personas que lo requieren, en éste sentido se ven reflejadas intervenciones y cuidados de las más alta calidad científica y humanística proporcionados a las personas y sus familias en el procesos de salud y/o enfermedad éste es el sentido de la profesión.

Si bien es cierto que en los últimos años la investigación su productividad y su aplicación han sido en el mundo entero motivos de transformación no sólo de una profesión, sino de la sociedad misma. En materia de salud, la aplicación de la investigación ha ido encaminada al descubrimientos de intervenciones oportunas de enfermería en el tratamientos para ciertas condiciones de salud y/o enfermedad así como de la prevención, promoción y bienestar de la salud; así, la investigación cobra mayor importancia al ser el proceso de sustentabilidad de la práctica clínica diaria, pues ésta arroja evidencias para guiar o planear los diferentes planes o intervenciones del cuidado que se debe otorgar.

En este sentido la metodología de investigación que utiliza el profesional de enfermería debe basarse en la implemen- 
tación del método científico basado en la mejor evidencia científica con la aplicación y rigor metodológico; ésta es la manera de hacer ciencia.

Actualmente la formación de los profesionistas de enfermería deberán basarse en el proceso de profesionalización, no obstante, estudios realizados demuestran que los planes de estudio existentes en diferentes países de centro y Latinoamérica no han considerado la formación investigativa en sus niveles básicos sino hasta los niveles de especialización y, en algunos otros, hasta el nivel de maestría, de tal manera que el impulso a la investigación en la disciplina desde el pregrado es débil; éste es un factor que está permeando en la escasez de publicaciones así como en la resolución de problemas en la práctica diaria.

Al respecto, la Organización Panamericana de la Salud señala que una de las principales barreras para la investigación en enfermería es la falta de conocimiento y la experiencia en el tema, así mismo en la discusión que se genera en su publicación establece tres líneas a desarrollar en el cumplimiento de la misión del profesional de enfermería, éstas son:

1. La investigación centrada en la persona.

2. El desarrollo de programas formales de investigación en enfermería.

3. El fomento a la investigación interdisciplinaria y multidisciplinaria. $^{21}$

\section{CONCLUSIÓN}

En las instituciones y centros de salud el reto es aún mayor, ya que el concepto de calidad de atención debe basarse en cuidados personalizados de cada enfermo, no obstante, la atención directa debe basarse en la mejor evidencia científica, el trato digno y en los estándares e indicadores actuales. Estos datos actualmente son medibles en cada enfermo y su familia.

El cuidado, actualmente, ha significando un elemento invaluable para investigar y mejorar la atención, al prevenir, planear y proporcionar en cada persona - bajo las condiciones de salud actuales - que se favorezcan la continuidad del mismo por los profesionales de enfermería a cargo de los diferentes servicios.

\section{REFERENCIAS}

1. Amaro-Cano MC. Florence Nightingale, la primera gran teórica de enfermería. Rev Cubana Enfermer. 2004; 20 (3): 2-3.

2. Espinoza-Ortega $\mathrm{S}$. Tendencias actuales de la investigación de enfermería en salud pública. VI Congreso Regional de Salud Pública Facultad de Enfermería, UANL.

3. Moreno-Casbas M. Boletín IDEA. Investigación, Docencia, Enfermera Avanzada. 2005; 4.

4. Calhoun JG, Davidson PL, Sinioris ME, Vincent ET, Griffith JR. Toward an understanding of competency identification and assessment in health care management. Quality Management in Health Care. 2002; 17 (1): 14-28.

5. Manfredi M. La investigación en enfermería en América Latina. Educación Médica y Salud. 1991; 25 (2): 154-166.

6. Organización Panamericana de Salud. Nuevos retos en el campo de la educación avanzada del personal de enfermería en América Latina. Pan American Journal of Public Health. 199; 2(1): 51-56.

7. Romero MN. Formación de Investigadores en enfermería. Conclusiones de Simposios. VII Conferencia Iberoamericana de Educación en Enfermería [Internet]. [acceso el $1^{\circ}$ de septiembre]. 2004. Disponible en: http://www.aladefe.org/indexfiles/docs/conclusiones_cidee/formacioninvestigadores_enfermeros.doc

8. Castrillón C. Trends and priorities in nursing research. Revista Latinoamericana Enfermagen. 2004; 12 (4): 583-588.

9. Araneda G. Proyecto especial MECESUP 2004 curricular ¿es el cambio que debemos tomar? ( $\left.\mathrm{N}^{\circ} 3\right)$. Santiago: Asociacion Chilena de Educación en Enfermería; 2004.

10. Registered Nurses Association of British Columbia. Nursing and Research Policy Statement (Report N 94).Vancouver: RNABC; 2003.

11. Consejo Internacional de Enfermeras. Declaración de posición: Investigación en Enfermería [Internet]. 1999 [Revisado el $1^{\circ}$ de septiembre, 2004]. Disponible en: http://www.icn.ch/psresearch99sp.htm

12. Lamelas EF. La investigación en el currículum de enfermería. Grado de influencia y límites de la enfermería basada en la evidencia. Enfermería Clínica. 2002; 1 (4): 182-187.

13. Registered Nurses Association of British Columbia. Nursing and Research Policy Statement (Report No 94). Vancouver: RNABC: 2003.

14. Op. Cit. Castrillón, p. 585.

15. Organización Panamericana de la Salud. Valores y competencias corporativas para el personal profesional en la Organización Panamericana de la Salud (Documento de síntesis). Washington, D.C.: Organización Panamericana de la Salud; 2001.

16. Op. Cit. Colhoun, p. 26.

17. Donaldson B. La investigación en el conocimiento y la práctica de enfermería [Internet]. Disponible en: www.sonico.com/...enfermeria/.../ la-investigacion-en-enfermeria-en-america-latina

18. Op. Cit. Espinoza-Ortega.

19. Harrison L, Hernández R. Competencias para Investigación en diferentes niveles de formación de enfermeras: Una perspectiva Latinoamericana. Cienc. Enferm. 2005; 11 (1).

20. Suing A. Definición de líneas de investigación. Compilación para insumos CITTES. España: Universidad técnica particular de Loja; 2008.

21. Stacciarini, Investigación en enfermería en Brasil: es el momento de un cambio.

22. Abel Suing. Establecimiento de líneas y temas de investigación. 